\title{
Bioanalysis
}

\section{The changing world of bioanalysis: summary of panel discussions}

First draft submitted: 30 March 2017; Accepted for publication: 15 May 2017; Published online: 1 August 2017

\section{Keywords: bioanalysis $\bullet$ outsourcing $\bullet$ pharma-CRO relationship $\bullet$ skills $\bullet$ training}

The world of bioanalysis is changing rapidly. The move toward the measurement of endogenous molecules to understand drug pharmacodynamics (PD), efficacy and toxicity and the expanding numbers of novel molecular constructs for pharmaceuticals (peptides, proteins, oligonucleotides, antibody-drug conjugates, gene therapy, etc.) bring exciting analytical challenges. Further, novel analytical instrumentation and workflows are continually being developed, offering benefits, new ways of working and bringing associated challenges (e.g., microsampling, high resolution mass spectrometry [HRMS], capillary separations, etc.). In addition to this, pharmaceutical companies (Pharma) are increasingly turning to outsourcing as a routine approach, requiring different ways of working. On top of this, the regulatory landscape is continually changing. All of these changes inevitably bring stimulating challenges and opportunities for current and future practitioners of bioanalysis, requiring them to continually develop themselves in order to stay current. In November 2016 at a venue in Barcelona, Bioanalysis Zone brought together eleven bioanalytical experts from Pharma, Contract Research Organizations (CROs) and consultancy companies to discuss these issues that are facing the quantitative bioanalytical community. The panel discussion was filmed and can be viewed full on the Bioanalysis Zone website [1]. The following is a summary of this insightful and honest dialogue, which was built on a similar event earlier in 2016 in Orlando [2,3].

\section{How do we access the required skill sets?}

The initial discussion centered around whether staff in quantitative bioanalytical laboratories in Pharma and CRO companies have the skills to equip them for the challenges arising from these novel analytical constructs, workflows and instrumentation, and consequently whether we are adequately training the next generation of bioanalytical scientists. It was agreed that to meet these analytical challenges, new skill sets are required including learning how to use and apply novel instrumentation, and to combine technologies and develop novel analytical approaches from what have traditionally been separate areas. It was noted that even for small molecule pharmaceuticals, the nature of the molecules is changing, with them becoming harder to analyze using standard LC-MS/MS approaches and that alternative methods such as derivatization and supercritical fluid chromatography are now often being required. Further, project teams are increasingly requiring bioanalytical scientists to have broader based scientific understanding of drug development for both small and large molecules in addition to their analytical contributions. This includes understanding the pharmacology and toxicology of the test molecule and close involvement in clinical trials.

It was considered that while the bioanalytical scientists have the tools and instrumentation they require to solve these emerging exciting analytical challenges, it is apparent that in general they are still acquiring the additional
Neil Spooner*,1,2, Melanie Anderson ${ }^{3}$, Lieve Dillen ${ }^{4}$, Luca Ferrari ${ }^{5}$, Martijn Hilhorst ${ }^{6}$, Zamas Lam7, Marco Michi $^{8}$, James Munday ${ }^{9}$, John Smeraglia' ${ }^{10}$, Scott Summerfield ${ }^{11}$ \& Dieter Zimmer ${ }^{12}$

'Spooner Bioanalytical Solutions Ltd, Hertford, SG13 8BQ, UK

${ }^{2} S$ chool of Life \& Medical Sciences, University of Hertfordshire, Hatfield, AL10 9AB, UK

${ }^{3}$ Department of Pharmacokinetics, Pharmacodynamics \& Drug Metabolism, Merck Research Labs, West Point, PA 19486, USA

${ }^{4}$ Development Bioanalysis, Janssen Research \& Development, Beerse, B-2340, Belgium

${ }^{5}$ Roche Innovation Center, Basel, $\mathrm{CH}-4070$, Switzerland

${ }^{6}$ Small Molecules Bioanalytical Laboratory, Early Development Services, PRA Health Sciences, Assen, 9407 TK,

The Netherlands

7QPS, LLC, Newark, DE 19711, USA ${ }^{8}$ ADMET \& Bioanalytical Sciences, Aptuit srl, Verona, 37135, Italy

${ }^{9}$ Covance, Bioanalysis (Europe), Otley Road, Harrogate, North Yorkshire, HG31PY, UK

${ }^{10}$ Bioanalytical Sciences, UCB BioPharam SA, chemin du Foriest, B-1420 Braine-

I'Alleud, Belgium

"Bioanalysis, Immunogenicity \& Biomarkers, GlaxoSmithKline Pharmaceuticals Research \&

Development, Park Road, Ware, SG12 ODP, UK

${ }^{12 Z i m m e r ~ B i o A n a l y t i c s ~ \& ~ M o r e, ~}$

4052 Basel, Switzerland

*Author for correspondence:

neil@spoonerbioanalytical.co.uk

\section{newlands} press part of 
skills and experiences they need to take on these challenges and best use these tools. There was discussion as to whether it is best for an organization to obtain these skills and experience through the development of their staff, or through hiring of suitably skilled staff. The consensus was that a combination of these approaches would work best. Training of existing staff offers the benefit of enriching staff roles and increasing motivation, leading to increased staff retention and development. It also builds upon the broader institutional knowledge, familiarity with the portfolio of a company, drug development skills and specific understanding of regulations and requirements around data quality that are often held by existing staff. It was noted that companies often have the required skills to approach these analytical problems, but that the experts are situated in other departments. Improved communication, cross-training and secondments were put forward as solutions to overcome this issue. On the other hand, recruiting new staff brings in fresh energy, ideas, techniques and technologies. However, when these new recruits come from outside the industry, they generally lack the broader understanding of drug development and the specifics of regulatory requirements, particularly for regulated quantitative bioanalysis.

It was noted that while there are benefits in recruiting new staff from universities, specific training in techniques such as HRMS and the background to assay performance, such as validation, precision and accuracy, among others, was often lacking in these institutions. In some cases, even technically skilled students have limited experience in accurate pipetting. PRA have observed that in The Netherlands there are no issues with the availability of good quality laboratory technicians, due to the presence of good laboratory schools and interest generated by television shows such as CSI. These students tend to have good technical skills when recruited by an operational lab and there are no issues with training them more broadly in other aspects of bioanalytical workflows. However, issues are observed with the recruitment of bioanalysts with higher level qualifications, such as $\mathrm{PhD}$, as there are few programs training bioanalysts at this level in The Netherlands. Covance have noticed a dearth in the availability of fully trained bioanalysts in the UK. Their response has been to sponsor a Master's degree program at the University of Leeds, where students are specifically trained in bioanalytical skills with the active involvement of Covance staff. One opinion was that staff should get their background in the general requirements for regulatory bioanalysis (such as GLP, validation, linearity, precision, accuracy, stability, among others) by being trained in small molecule bioanalysis first, before progressing onto the requirements of biopharmaceutical bioanalysis.
One of the difficulties in recruiting and retaining staff in bioanalytical groups was the perception of the discipline not being 'sexy' compared with other scientific and nonscientific career possibilities. It was agreed that there is much to be done in the marketing of regulated quantitative bioanalysis in order to improve this situation. It was expressed that the increasingly diverse opportunities brought about by the new molecular constructs, novel analytical approaches and the growing involvement of bioanalytical scientists in broader drug development will all help in this respect. Further, encouraging the career development through involvement in university teaching programs and registration for $\mathrm{PhDs}$ were seen to be positive ways forward.

\section{What will the bioanalytical lab of the future look like \& what skills will this require?}

The conversation then continued by exploring how drug development and, therefore, the bioanalytical laboratory might change in the future and, hence, what the requirements might be for bioanalytical skills. It was agreed that the current trend of biopharmaceuticals and novel constructs composing a large proportion of the drug development portfolio for Pharma will continue. In turn, this will strengthen the requirement for new analytical and drug development skill sets for bioanalysts of the future. Further, bioanalysts will increasingly require knowledge of molecular biology and biochemistry in order to work with these novel constructs and to assist with understanding the PD and pharmacology of candidate drugs.

This increase in novel pharmaceutical constructs will increasingly require bioanalysts to have a blend of analytical skills from what have previously been seen to be separate groups in most Pharma and CRO organizations. Those with traditional strengths in analytical chemistry-based small molecule LC-MS approaches will increasingly develop skills in biological analytical approaches and a knowledge of biology and pharmacology. Conversely, those who have worked in more biologically based fields utilizing immunoassays will develop skills in analytical chemistry, chromatography, MS and in the regulatory requirements for drug development and the registration of a new medicine. Both groups will need to develop their skills in other approaches not currently commonly found in either, for example, HRMS. This requirement for the cross-fertilization of skills will inevitably strengthen the links between the currently disparate chemical and biology-based analytical groups. It was generally agreed that the future of bioanalysis will not be separate small and large molecule bioanalytical units, but an integrated department having the skills and instrumentation to be able to deal with both, and for other analytical challenges that will inevitably arise. 
A number of other observations on how bioanalytical groups may develop in the future were also made. For example, the growing utilization of HRMS, will require staff to develop their analytical skills. However, there were differing opinions as to how this could be achieved, either via specialized training, or through on the job training and reading the scientific literature. The increasing development of therapeutics based upon polynucleic acids was seen to offer a unique analytical challenge, as most companies do not currently have the skill sets. Another observation was that bioanalytical groups are likely to increasingly focus on the analysis of biomarkers and other endogenous molecules. In addition, it was noted that bioanalytical groups may have a greater responsibility for the qualitative aspects of analysis (metabolite and impurity identification and detection), in addition to their quantitative responsibilities. While developing their analytical skills, it was also considered that bioanalysts will need to increase their understanding and involvement in other aspects of drug development. This may include an increasing involvement in the deployment of novel techniques used to collect samples, the interpretation of the bioanalytical data they generate and developing in-depth knowledge of the increasingly diverse therapeutic areas and pharmacology being explored by Pharma to be able to put that data in context.

An important consideration was how does bringing these different parts of Pharma together affect the outsourcing model. Are the necessary skills and equipment available at CROs? It was apparent that CROs are closely following what Pharma is doing, and are building the required skills and buying the necessary instrumentation. However, it was stated that it is more difficult for the CRO to be closely involved with the biology of a particular project and interpreting the data in the broader context of drug development, as they are a step removed from that process. It was noted that CROs are definitely moving to bridge this gap and are increasingly good at building complex packages of data, with immunogenicity being put forward as a good example. In this case, CROs will develop assays for the drug, for antidrug antibodies and for PD markers. They will then pull together the datasets to interpret what is happening with immunogenicity. However, this will require good CRO/ Pharma collaboration to enable this to happen.

It was noted that whilst it is challenging, CROs are also adept at varying their models of how to work between the requirements of different clients. The working relationship between CROs and Pharma was considered to be dependent on the amount of experience in bioanalysis of the Pharma partner. Some clients have very little bioanalytical expertise and are happy to be hands-off with all aspects of assay development, application and understanding of the data. Other Pharma companies may have a more active oversight through study monitors, who may be able to offer advice on a particular project, but are unable to offer lab-based skills and understanding. Alternatively, other Pharma companies may have an active internal bioanalytical group who are able to develop complex assays, or offer advice to CROs when difficulties arise. In many cases, these internal Pharma bioanalytical groups will keep what are perceived to be tricky assays in-house and only outsource them when they become routine.

It was observed that historically, individuals with bioanalytical skills have tended to stay with either Pharma, or CRO businesses for most of their career. However, there now appears to be more bi-directional movement between the two, which inevitably brings a cross-fertilization of ideas, technologies and working practices.

There was an in-depth discussion on the current and future role of automation in quantitative bioanalysis with a wide range of views being expressed. The potential benefits are well understood and include increased assay reproducibility, increased data integrity, reducing the potential for sample contamination and freeing-up staff to perform other activities, including data interpretation and closer scientific involvement with the drug development project. However, whilst many companies understand the potential benefits of implementing more automation into their bioanalytical workflows and have invested in equipment, few consider that they have fully realized those benefits. The reasons for this include, a failure to convince analytical staff that their data will not be adversely affected by automation and a perception that increasing automation puts the scientists role at risk. In addition, it was considered that the increased outsourcing of the bioanalysis of samples derived from large clinical studies by Pharma has resulted in samples from only smaller clinical and pre-clinical studies being available for in-house analysis. The smaller sample numbers available from these studies make the benefits of applying automation less obvious to Pharma, as it is considered that fewer efficiencies can be achieved. In addition, contributors considered current automation approaches to be inflexible and best suited to standardized processes, making it difficult to adapt workflows for difficult analytical challenges. Further, it was noted that differing levels of programming expertise are required, depending on the level of automation. This can require significant investment.

This led to a discussion as to whether CROs should be taking the lead in developing automation approaches as they are the ones predominantly supporting large clinical studies where it is perceived that the greatest benefits can be obtained. However, on closer inspection it was apparent that the CROs also need to consider the 
benefits of task-based versus total automation. They are working for multiple sponsors with different numbers of samples, tube types and assay requirements, among others. Further, it was stated that with current approaches, sample processing and analysis is not the bottleneck, for example, it was considered that a study with 1000 samples can be processed manually just as fast as it can be if automated. Rather, data processing and reviewing were perceived to be a major hold-up. However, although automation for these purposes has been available for some time, for example, electronic laboratory notebooks (eLNB), they are not widely used. It was stated that it can be difficult for CROs to implement eLNBs, as they have clients who want things doing in different ways and who may have concerns around their data being processed and stored in this way. However, the feedback from the participants with a Pharma background did not confirm this. It was recognized that eLNB has the potential to significantly increase efficiency and data transparency, as documentation is stored in one location. As the industry sees more mobility in its workforce, this can contribute to the seamless transfer of responsibilities and institutional knowledge. Another hurdle to the implementation of eLNBs and automation in general was the requirement for GLP-compliant systems. There is a large overhead in validating systems in such environments. Further, it requires changes to established auditing processes, with QA departments and external auditors requiring access to electronic data and training. In addition, where eLNB systems are implemented, bioanalytical groups will need to develop expertise in this technology to select the best systems and maintain them to current regulatory practice. It was notable that several of the Pharma representatives stated that they had successful implemented eLNBs, particularly for discovery workflows where it has very much helped with data integrity. However, the implementation burden was considered to be lower than that in a fully regulated environment.

Based upon this broadly held experience, the consensus was that automating certain parts of the bioanalytical process, rather than the whole workflow, is more likely to occur successfully and realize some of the recognized benefits. This could be for sample aliquotting, sample labeling, etc. Further, there was a consensus that the management of bioanalytical groups need to understand that this type of change will not happen in a year or two. It will need a lot of time, investment, knowledge gathering and support. Ideally, a group implementing automation will build a degree of expertise in the systems for themselves. This will minimize the possibility of the automation being used inappropriately and therefore generating incorrect data, that is, using the instrumentation to dilute samples with plasma, when the liquid handler is calibrated for water. The suggested stepwise approach then builds trust with the bioanalyst. They can see that they have a companion that will help them deliver their work more efficiently and is not a threat to their role. It will also help them to build trust compared with working with a larger automated system. It is hoped that bioanalysts will start to see that automation is in fact freeing-up their time, enabling them to become experts in their existing skills and/or add others as discussed earlier.

It was noted that many ligand binding workflows already have a high degree of automation, for example Gyrolab $^{\mathrm{TM}}$ systems [4]. The participants considered that this technology works well for large clinical study and/ or for a study with limited sample volume. However, it may not offer the same advantages for other types of study.

\section{Embedding science in the Pharma-CRO relationship}

The developing trend in the relationship between Pharma and CROs was increasingly seen to be one of scientific partnership, with both parties considering the other as equals, rather than the historical transactional model. However, the nature of this relationship varies, depending on the needs of the client. For example, small Biotech companies tend to have less in-house bioanalytical and drug development expertise and so will require more scientific input from the CRO. Whereas, a large Pharma that has a lot of its own expertise, will generally drive the scientific relationship. However, it is also becoming apparent that as CROs interact with, and learn from, numerous clients and encounter a diversity of molecular and analytical challenges, they are becoming the go-to experts in bioanalysis, rather than the historical position of Pharma groups leading in this specialty.

One observation was that scientist to scientist conversations between the client and CRO are often straightforward, particularly when smaller biotech companies are involved. These companies have little, or no in-house bioanalytical expertise and so inevitably have to rely on that of the CRO. However, the relationship between large Pharma and CROs is often much more complex. While scientist to scientist communication still happens successfully, other requirements begin to become apparent. In particular, the increasing involvement of sourcing groups in negotiating pricing and deliverables as master service agreements, tends to downplay the scientist to scientist interaction and make the relationship more transactional. In turn, this has led to the outsourcing of bioanalytical work increasingly being seen as a commodity by leaders in Pharma companies. Inevitably, this can result in a disconnect between the bioanalytical scientist and procurement groups and can create a communica- 
tion challenge. For example, the scientist (at the Pharma and/or CRO) may say that this particular assay is difficult and will, therefore, require more work and a different cost base and time frame. It was stated that the response to this from procurement groups will often be that this is a commodity and should be paid for and delivered at the agreed price and time frame as set out in the master service agreement. Further, it was stated that there is an increasing expectation that method development and validation of a method are provided for free by the CRO. It was agreed that to overcome these issues, scientific decisions need to be able to take place and that the relationship between Pharma and CRO needs to grow and mature to enable realistic conversations to occur. It was suggested that bioanalytical scientists need to take responsibility to work with procurement groups and their own management to help them understand that some assays will take longer and therefore cost more, and that not every assay fits into the simple commoditybased model. This is especially true in the current more challenging environment with more complex small and large molecules.

Another observation was that the recent move toward the downsizing of Pharma bioanalytical groups has led to a shift in the Pharma/CRO relationship. As Pharma companies often have less internal bioanalytical skills, they are becoming increasingly reliant on those of the CROs. This is resulting in CROs not only developing bioanalytical methods and analyzing study samples, but increasingly taking a lead in the development of novel approaches, technologies, the training/recruitment of staff with the required skills and the provision of the required analytical capacity. Further, the CROs are increasingly advancing their more general drug development expertise. The relationship is now more one of equals, rather than a master/servant agreement that it was in the past. This is exemplified by the relationship with smaller Biotechs companies where often the CROs are routinely acting as a consultant to help them progress their compound from candidate selection through to FTiH studies. However, some concerns were expressed that as large Pharma bioanalytical groups continue to downsize, there may be a lack of later phase project knowledge within the Pharma company to pass on to the CROs. Examples included the

\section{References}

1 Spooner N, Anderson M, Dillen L et al. The Changing World of Bioanalysis Panel Discussion, Barcelona, Spain (2016). www.bioanalysis-zone.com/2017/02/01/cwb_spo/

2 Spooner N, Cape S, Hayes R et al. Issues facing the bioanalytical community: summary of round table discussions. Bioanalysis 8(21), 2189-2193 (2016). potential requirement for monitoring an active metabolite. It was also noted that the relationships between Client and CRO are likely to be different for small and large molecule work. For large molecule bioanalysis the scientists also need to understand immunogenicity and its physiological impact (PD), in addition to the analytical aspects. Therefore, interactions across disciplines both within the client and the CRO companies is also important. This is in-turn changing how CROs structure themselves and the skills they need to develop, or recruit in their workforce, to further enable the CRO to be regarded as an extension and partner of the Pharma company. For example, many CROs now have scientific director roles with a responsibility to get involved directly in broader scientific discussions. In some cases these individuals from the CRO sit on the Pharma clinical teams.

\section{Conclusion}

Numerous issues and challenges were discussed by the panel and it was apparent that the discipline of quantitative bioanalysis will change substantially in the future. However, it was clear that there are many ways forward to not only deal with the issues, but to have a clear positive impact on the career choices and job variety of quantitative bioanalytical scientists and enable the support of future drug development programs to deliver new medicines for patients.

\section{Acknowledgements}

The authors thank Sarah Bishop and Sankeetha Nadarajah (employees of Future Science Group) for facilitating the round table discussion and helping to make it such a positive and enjoyable event.

\section{Financial \& competing interests disclosure}

$\mathrm{N}$ Spooner took part in and chaired the Panel Discussion in the capacity of Senior Editor of the journal Bioanalysis. All expenses for his trip were covered by Future Science Group. The authors have no other relevant affiliations or financial involvement with any organization, or entity with a financial interest in or financial conflict with the subject matter or materials discussed in the manuscript apart from those disclosed.

No writing assistance was utilized in the production of this manuscript.

3 Spooner N, Cape S, Hayes R et al. Bioanalysis Zone Panel Discussion, Orlando, USA (2016).

www.bioanalysis-zone.com/2016/06/28/bioanalysis

4 Gyros company webpage. www.gyros.com/ 